\title{
A first step in reconstructing the solar corona self-consistently with a magnetohydrostatic model during solar activity minimum
}

\author{
P. Ruan ${ }^{1}$, T. Wiegelmann ${ }^{1}$, B. Inhester ${ }^{1}$, T. Neukirch ${ }^{2}$, S. K. Solanki ${ }^{1}$, and L. Feng ${ }^{1}$
}

1 Max-Planck-Institut für Sonnensystemforschung, Max-Planck-Strasse 2, 37191 Katlenburg-Lindau, Germany e-mail: ruan@linmpi.mpg.de

2 School of Mathematics and Statistics, University of St. Andrews, St. Andrews KY16 9SS, UK

e-mail: thomas@mcs.st-and.ac.uk

Received 11 October 2007 / Accepted 30 November 2007

\begin{abstract}
Aims. We compute the distribution of the magnetic field and the plasma in the global corona with a self-consistent magnetohydrostatic (MHS) model.

Methods. Because direct measurements of the solar coronal magnetic field and plasma are extremely difficult and inaccurate, we use a modeling approach based on observational quantities, e.g. the measured photospheric magnetic field, to reconstruct the structure of the global solar corona. We take an analytic magnetohydrostatic model to extrapolate the magnetic field in the corona from photospheric magnetic field measurement. In the model, the electric current density can be decomposed into two components: one component is aligned with the magnetic field lines, whereas the other component flows in spherical shells. The second component of the current produces finite Lorentz forces that are balanced by the pressure gradient and the gravity force. We derive the 3D distribution of the magnetic field and plasma self-consistently in one model. The boundary conditions are given by a synoptic magnetogram on the inner boundary and by a source surface model at the outer boundary.

Results. The density in the model is higher in the equatorial plane than in the polar region. We compare the magnetic field distribution of our model with potential and force-free field models for the same boundary conditions and find that our model differs noticeably from both. We discuss how to apply the model and how to improve it.
\end{abstract}

Key words. Sun: corona - Sun: magnetic fields - magnetohydrodynamics (MHD)

\section{Introduction}

To understand the physical processes in the solar corona (e.g. flares and coronal mass ejections), it is important to obtain information about the magnetic field that couples the solar interior with the atmosphere. The magnetic field on the Sun's surface is usually measured through the Zeeman effect displayed by magnetically sensitive spectral lines. Maps of the line-of-sight magnetic field (magnetogram) have been regularly recorded for numerous years, and more recently vector magnetograms have begun to be reliably recorded. In the corona, however, it is more difficult to measure the magnetic field directly. Measurements in the radio range allow the magnetic field strength to be determined (White 2002), but not the full magnetic field vector.

Other techniques are also available (see Lagg 2005, for a review), but also have their shortcomings. An alternative way of determining the coronal magnetic field is to extrapolate from photospheric measurements. The extrapolation result depends on the assumptions made regarding the coronal plasma and, in particular, the electric current density. The simplest approach for an extrapolation is a current-free potential field, which has been widely used to extrapolate the coronal magnetic field in the past (see e.g. Schmidt 1964; Semel 1967; Hoeksema 1991). In these potential models, the current-free assumption is assumed between the photosphere and the source surface. Although these models are easy to construct and have demonstrated some success in reproducing large long-lived structures, details of magnetic structure are often not approximated well by these models, particularly in active regions, (see e.g. Schrijver et al. 2005; Wiegelmann et al. 2005b). A more advanced approach is to construct force-free models, which contain electric currents along field lines. A subclass of these force-free models are the linear force-free field (LFFF) models (e.g. Chiu \& Hilton 1977; Seehafer 1978) where the current is parallel to the magnetic field with a global constant of proportionality $\alpha$ between current and field. For certain types of boundary conditions, the linear forcefree field is not uniquely determined; the same applies to the MHS solutions used in this paper.

The extrapolation with such LFFF models from a photospheric manetogram has been shown to explain many of the observed features of filaments (see, e.g., Aulanier \& Demoulin 1998; Aulanier et al. 2000). The force-free parameter $\alpha$ in LFFF models has been computed by comparing extrapolated magnetic field lines with coronal EUV-images (e.g. Carcedo et al. 2003; Marsch et al. 2004; Wiegelmann et al. 2005a). The LFFF models have also been used in combination with images from different viewpoints to stabilize the stereoscopic reconstruction of 3D coronal loop structures by Wiegelmann \& Neukirch (2002); Wiegelmann \& Inhester (2006) and Feng et al. (2007). An advantage of LFFF models is that they only require photospheric line-of-sight magnetic field observations as input, but they have a free parameter and are not as accurate as the more 
sophisticated approaches like nonlinear force-free field (NLFFF) models, which is the generic case for force-free fields. These models are mathematically more challenging and require measurements of the photospheric magnetic field vector as input (see e.g. Aly 1989; Sakurai 1989; Amari et al. 1997; Wheatland et al. 2000; Wiegelmann 2004; Schrijver et al. 2006; Inhester \& Wiegelmann 2006; Amari et al. 2006; Wiegelmann 2008; Metcalf et al. 2008). Direct measurements of the magnetic field in an active region by Solanki et al. (2003) have been compared with extrapolations under different model assumptions by Wiegelmann et al. (2005b). The study revealed that the LFFF model is better than the potential field model, but is not as accurate as the NLFFF model.

The main reason for the success of force-free field model extrapolation is the low plasma $\beta$ in the low corona, which means the magnetic field is the dominating quantity and plasma carried by the magnetic field has little influence on the field. However, from force-free models, we cannot derive the plasma density and pressure directly because all the plasma information is totally ignored in the models. Consequently, FFF models cannot by themselves predict coronal emission, which is important for the comparison with EUV and X-ray measurements. To circumvent this deficiency, scaling laws have been used to model the coronal plasma along magnetic loops (see, e.g., Rosner et al. 1978; Serio et al. 1981; Kano \& Tsuneta 1995; Aschwanden et al. 2000). These methods - modelling first the coronal magnetic field and thereafter the plasma along the field lines - have been successfully applied to global potential fields by Schrijver et al. (2004) and obtained reasonable agreement with observed plasma images. However, we cannot consider these models as completely satisfactory because they are not self-consistent.

Complementary to these reconstructions based on observational data, self-consistent modelling approaches using magnetohydrostatics and magnetohydrodynamics have been developed (see, e.g., Pneuman \& Kopp 1971; Cuperman et al. 1992; Wu et al. 1995, 1997; Wiegelmann et al. 1998; Antiochos et al. 1999; Wiegelmann et al. 2000). Theses models aim to describe coronal structures like helmet streamers and their association with coronal mass ejections and the solar wind. Limited by the computer resources of the time, these models were two-dimensional and describe observed coronal structures only qualitatively correctly. Despite these simplifications, such self-consistent models have some success in revealing possible mechanisms for the occurrence of coronal eruptions as observed in the early days of SOHO by Schwenn et al. (1997) during the solar activity minimum. Three-dimensional models of the corona using large-scale MHD simulations have, for example, been developed by Mikić et al. (1999).

In the present paper we aim to use three-dimensional, selfconsistent analytical MHS solutions as a basis for a model of the global corona. In spite of the general difficulties associated with finding three-dimensional analytical solutions of the MHS equations, some useful solutions are available (Low 1985; Bogdan \& Low 1986; Low 1991, 1992, 1993a,b; Neukirch 1995, 1997; Neukirch \& Rastätter 1999; Petrie \& Neukirch 2000). This class of solutions has a volume current density that flows in surfaces perpendicular to the direction of the gravitational force and a possible additional field-aligned current density. For models of the global corona, solutions in spherical coordinates as given by Bogdan \& Low (1986) and Neukirch (1995) are particularly useful. The solutions of Neukirch (1995) are based on the previous work by Bogdan \& Low (1986) and differ from them only by the additional field-aligned current density component. The fundamental equation in this MHS model is a Schrödinger type equation for the radial field component (for a simpler method see Neukirch \& Rastätter 1999). The Bogdan \& Low (1986) solutions have been discussed or used as a basis for global coronal models, for example, by Zhao \& Hoeksema (1993); Zhao \& Hoeksema (1994); Gibson \& Bagenal (1995), \& Gibson et al. (1996), whereas the Neukirch (1995) solutions have been discussed for coronal models by Zhao et al. (2000), and Rudenko (2001).

In this paper we use the (Neukirch 1995, subsequently referred to as N95) MHS solutions to develop a global coronal model using photospheric magnetograms as input for the magnetic field. We are aiming for a model that can be compared directly with observations and that describes the coronal magnetic field and plasma self-consistently.

The paper is organized as follows. We show how to apply the N95 MHS model to the construction of the magnetic field in the solar corona in Sect. 2. In Sect. 3, we explain how to get the plasma distribution in the corona. In Sect. 4, we show the result of field lines in the whole corona and the density in the source surface. The paper concludes with a summary and an outlook in Sect. 5

\section{Magnetic field}

In this section, we summarize Neukirch (1995)'s MHS model and introduce a modification to the model for its application to the solar corona. More details of the model can be obtained in $\mathrm{N} 95$.

The basis for the MHS model consists of the MHS equations:

$$
\begin{aligned}
\boldsymbol{j} \times \boldsymbol{B}-\nabla p-\rho \nabla \psi & =0, \\
\nabla \times \boldsymbol{B} & =\mu_{0} \boldsymbol{j}, \\
\nabla \cdot \boldsymbol{B} & =0 .
\end{aligned}
$$

Here, $\boldsymbol{j}$ and $\boldsymbol{B}$ are the electric current and the magnetic field, respectively, $p$ is the plasma pressure, $\rho$ the plasma density, $\psi$ the gravitational potential, and $\mu_{0}$ the permeability of vacuum.

In N95 the electric current was decomposed into two components:

$\mu_{0} \boldsymbol{j}=\alpha \boldsymbol{B}+\varepsilon(r)[\nabla(\boldsymbol{r} \cdot \boldsymbol{B})] \times \boldsymbol{r}$,

where $\varepsilon(r)=1 / r^{2}-1 /(r+a)^{2}$, which is a special choice in our model. Both $\alpha$ and $a$ are two free parameters in this model that are globally constant. The first term describes the field-aligned $j$, the second a toroidal $j$ that produces the Lorentz force to compensate for pressure gradient and gravity.

Inserting Eq. (4) into Eq. (2), taking the curl of Eq. (2) and dotting the resulting equation with $r$, they obtained

$\Delta(\boldsymbol{r} \cdot \boldsymbol{B})+\mu_{0} \varepsilon(r) \boldsymbol{L}^{2}(\boldsymbol{r} \cdot \boldsymbol{B})+\left(\mu_{0} \alpha\right)^{2}(\boldsymbol{r} \cdot \boldsymbol{B})=0$,

where $\boldsymbol{L}$ is the angular momentum operator,

$\boldsymbol{L}=\frac{1}{i} \boldsymbol{r} \times \nabla$

and $i$ is the imaginary unit.

By expanding $\boldsymbol{r} \cdot \boldsymbol{B}$ into a series of spherical harmonics,

$\boldsymbol{r} \cdot \boldsymbol{B}=\sum_{l=1}^{\infty} \sum_{m=-l}^{l} l(l+1)\left(\sum_{j=1}^{2} A_{l m}^{(j)} u_{l}^{(j)}(r)\right) Y_{l}^{m}(\theta, \phi)$ 


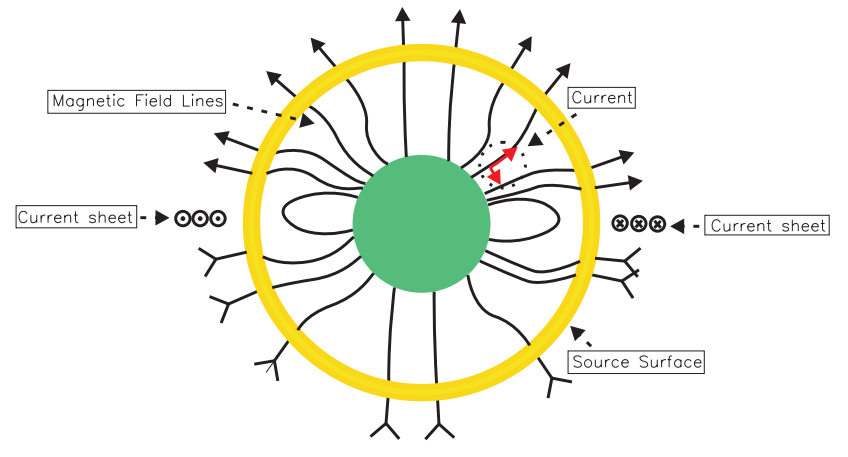

Fig. 1. Schematic representation of the MHS model.

and with the help of Eq. (3), N95 obtained an explicit expression for the magnetic field:

$$
\begin{array}{r}
\boldsymbol{B}=\sum_{l=1}^{\infty} \sum_{m=-l}^{l} \sum_{j=1}^{2} A_{l m}^{(j)}\left\{l(l+1) u_{l}^{(j)}(r) \frac{\boldsymbol{r}}{r^{2}} Y_{l}^{m}(\theta, \phi)\right. \\
+\frac{\mu_{0} \alpha}{i} u_{l}^{(j)}(r) \boldsymbol{L} Y_{l}^{m}(\theta, \phi) \\
\left.+\frac{\mathrm{d}\left(r u_{l}^{(j)}\right)}{\mathrm{d} r} \Delta Y_{l}^{m}(\theta, \phi)\right\} \\
\left(\begin{array}{c}
u_{l}^{(1)}(r) \\
u_{l}^{(2)}(r)
\end{array}\right)=\frac{\sqrt{r+a}}{r}\left(\begin{array}{c}
J_{l+1 / 2}(\alpha(r+a)) \\
N_{l+1 / 2}(\alpha(r+a))
\end{array}\right) .
\end{array}
$$

In Eq. (8) $l$ starts from 1 not 0 in order to rule out a magnetic monopole term. The variable $A_{l m}^{(j)}$ are a priori unknown coefficients to be determined from the boundary condition supplied by the photospheric magnetogram. Both $J_{l+1 / 2}(\alpha(r+a))$ and $N_{l+1 / 2}(\alpha(r+a))$ are Bessel functions of the first and the second kinds, respectively.

Two components of the current density are present in Eq. (4). The first term of the current $\alpha \boldsymbol{B}$ is the force-free part, and it cannot produce a Lorenz force on the plasma because it is parallel to the field lines. The second term $\varepsilon(r) \nabla(\boldsymbol{r} \cdot \boldsymbol{B}) \times \boldsymbol{r}$ is perpendicular to the radial direction, i.e. It flows on spherical surfaces. This current density component produces a Lorenz force which balances the pressure gradient $\nabla p$ and the gravity force $\rho \nabla \psi$ in Eq. (1). In our application to the solar corona, we take both the first and second kinds of Bessel function as given in Eq. (9). N95 took only the second kind of Bessel functions and presented explicit solutions only for spherical harmonics with $n=1$ and $n=2$ to generalize the Bogdan \& Low (1986) model.

Figure 1 shows a schematic illustration of our model. We place the source surface at 2.5 solar radii. Between the photosphere and the source surface, our model is given by the MHS model of N95. Outside of this boundary the magnetic field lines are purely radial. We have a toroidal current according to the variation in the magnitude of the magnetic field. This current is strongest near the equatorial plane where the radial component of the large-scale magnetic field reverses its sign.

In panel a of Fig. 2, we plot the synoptic photospheric chart for Carrington rotation 1919 (from Feb. 1, to Mar. 1, 1997) in the solar minimum of the radial magnetic field obtained from the Wilcox Solar Observatory, which is our boundary condition. The radial field is obtained by dividing the measured longitudinal magnetic field signal, $B_{\mathrm{LoS}}$, by $\mu=\cos \theta$, where $\theta$ is the heliocentric angle. Note that, because of the reduced spatial resolution at higher latitude introduced by foreshortening, magnetic flux is underestimated there, in particular in regions of mixed magnetic polarity (Krivova \& Solanki 2004). The resolution of this observation is 72 by 30 in the whole photosphere. The following panels display the radial field resulting from the MHS model with different numbers of spherical harmonics at 1 solar radius. When we take only five spherical harmonics $\left(N_{\max }=5\right)$, the model can give the general distribution of the magnetic field (the panel $b$ ). If we increase $N_{\max }$ to 10 and 20, increasingly smaller structures become visible; see panels c and d. For $N_{\max }=30$, the model output is very similar to the observation (panel e). If we take 40 spherical harmonics, the numerical noise in the model starts having a visible impact and produces structure especially in the polar regions (panel f), so we specify $N_{\max }=30$ for the following calculation.

\section{Plasma}

In the following we compute the plasma structure selfconsistently. Equation (1) can be decomposed into the following two equations:

$$
\begin{aligned}
\boldsymbol{j} \times \boldsymbol{B}-\nabla p_{\mathrm{pb}}-\rho_{\mathrm{pb}} \nabla \psi & =0, \\
-\nabla p_{\text {bg }}-\rho_{\mathrm{bg}} \nabla \psi & =0 .
\end{aligned}
$$

Here $p_{\text {bg }}$ and $\rho_{\text {bg }}$ are the background plasma pressure and density, respectively. They are homogenous in the corona and only functions of $r$. The $p_{\mathrm{pb}}$ and $\rho_{\mathrm{pb}}$ are perturbations of pressure and density produced by the magnetic field. They are the functions of $r, \theta, \phi$. According to Eq. (10), the Lorentz force $\boldsymbol{j} \times \boldsymbol{B}$ will cause the perturbation in the plasma and is balanced by the pressure gradient and the gravity. Eq. (11) describes that the background plasma balances itself without the magnetic field.

The total pressure and density functions are the sum of the perturbation part and the background part:

$p(r, \theta, \phi)=p_{\mathrm{bg}}(r)+p_{\mathrm{pb}}(r, \theta, \phi)$,

$\rho(r, \theta, \phi)=\rho_{\mathrm{bg}}(r)+\rho_{\mathrm{pb}}(r, \theta, \phi)$.

The plasma temperature can be calculated by

$T=\frac{m p}{\rho k_{\mathrm{B}}}$

where $\rho$ the mass density and $k_{\mathrm{B}}$ is the Boltzmann constant.

\subsection{Perturbation part of the plasma}

By inserting Eq. (4) into Eq. (1), one obtains explicit expressions of $p_{\mathrm{pb}}$ and $\rho_{\mathrm{pb}}$ in terms of $\boldsymbol{B}$ and $\boldsymbol{r}$ after some mathematics (detail see in N95):

$p_{\mathrm{pb}}(r, \theta, \phi)=-\frac{1}{2} \varepsilon(r)(\boldsymbol{r} \cdot \boldsymbol{B})^{2}$,

$\rho_{\mathrm{pb}}(r, \theta, \phi)=\frac{r^{2}}{G M}\left(\frac{1}{2} \frac{\mathrm{d} \varepsilon(r)}{\mathrm{d} r}(\boldsymbol{r} \cdot \boldsymbol{B})^{2}+r \varepsilon \boldsymbol{B} \cdot \nabla(\boldsymbol{r} \cdot \boldsymbol{B})\right)$.

Equation (15) implies that the perturbation pressure can be calculated analytically, whereas the perturbation density must be calculated numerically from Eq. (16). To determine the numerical grid appropriate for 30 spherical harmonics, we compute the $\rho_{\mathrm{pb}}$ independently from Eqs. (16) and (10). For Eq. (10) we calculate the perturbation pressure by Eq. (15) first. By comparing the densities from these two different methods, we check the accuracy of our calculation. We define the error $I$ as

$I=\left(\frac{1}{N} \sum_{k=1}^{n} \frac{\left|\rho_{\mathrm{pb}(k)}^{(10)}-\rho_{\mathrm{pb}(k)}^{(16)}\right|}{\rho_{\mathrm{pb}(k)}^{(10)}}\right)$, 


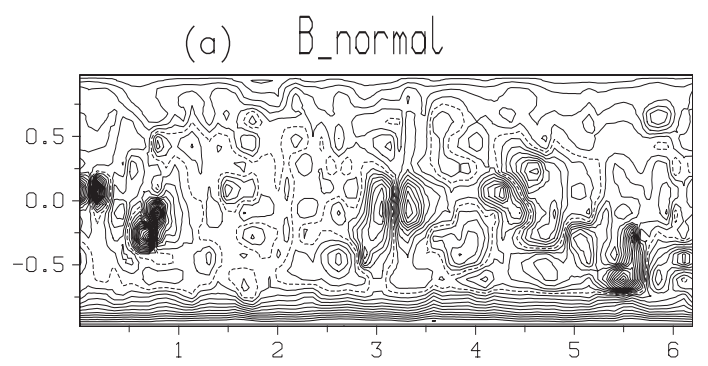

(b)

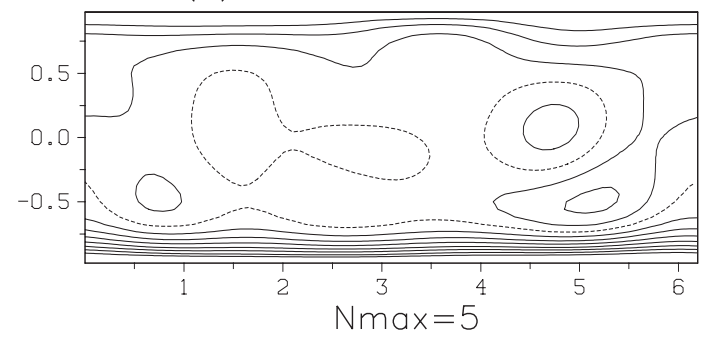

(c)

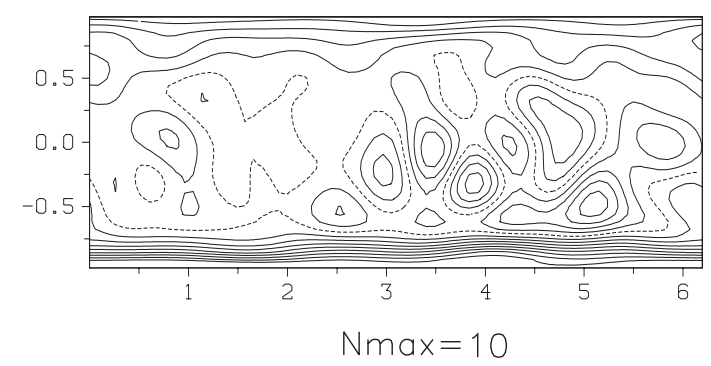

(d)

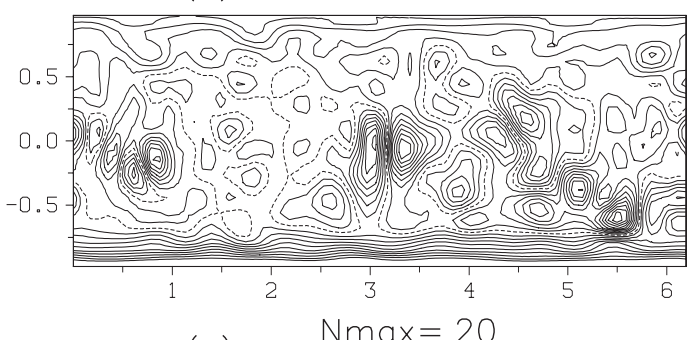

(e)

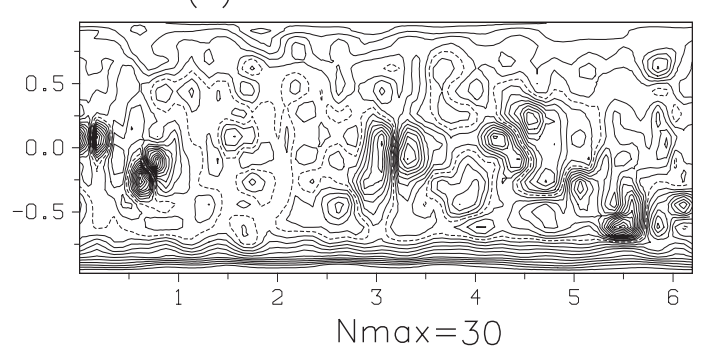

(f)

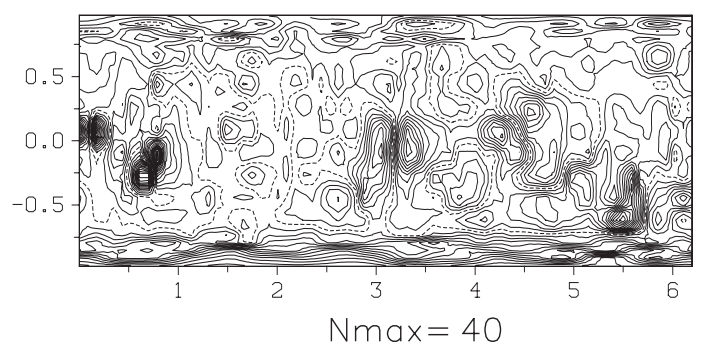

Fig. 2. Radial component of the synoptic photospheric magnetic field for Carrington rotation 1919 (the panel a)) and the radial component of the magnetic field from the MHS model with different numbers of spherical harmonics $\left(N_{\max }\right)$. The $x$-axis contains the longitude from 0 to $2 \pi$ and the $y$-axis the sin of the latitude.

where $\rho_{\mathrm{pb}(k)}^{(10)}$ and $\rho_{\mathrm{pb}(k)}^{(16)}$ denote the perturbation densities at grid points derived from Eqs. (10) and (16), respectively. There, $N$ is the total number of the grid points in the entire computational domain. We find that $I$ decreases to less than $4 \%$ when we put 80 grid points in the radial direction, 180 grid points in the $\theta$ direction, and 360 grid points in the $\phi$ direction for 30 spherical harmonics. This means the result of our calculations is consistent except for a small discretization error.

From Eqs. (15) and (16), we find that the perturbation pressure is always negative and the perturbation density could be negative in some places. Our model shows the strongest perturbation is always negative and located in the lower coronal layers of active regions. This imposes a requirement to insert a background plasma to compensate for the negative perturbation plasma density.

\subsection{Background plasma}

We use Eq. (11) to compute the background plasma as a stratified atmosphere. We also need the equation of state:

$p_{\text {bg }}=2 n_{\text {bg }} k T_{\text {bg }}$

where $T_{\mathrm{bg}}$ and $n_{\mathrm{bg}}$ are the background temperature and electron number density. Consequently, for the background, we have two equations (Eqs. (11) and (18)) and three variables: temperature, pressure and density. We can make assumptions for one variable (like the density) and calculate the other two variables from the equations.
On the one hand, as mentioned above, the perturbation density could be negative so that we need to apply a large enough background density in order to make the total density, which is the sum of the perturbation part and the background part, positive. On the other hand, we cannot make the background density too high. The background plasma is only a function of $r$. If the background is too large compared with the perturbation part, the total plasma will also be almost homogeneous, which certainly does not agree with observations.

The Baumbach-Allen formula gives a reasonable background of the plasma density distribution in the corona:

$n_{\mathrm{bg}}(r)=10^{8}\left[2.99\left(\frac{r}{r_{\odot}}\right)^{-16}+1.55\left(\frac{r}{r_{\odot}}\right)^{-6}+0.036\left(\frac{r}{r_{\odot}}\right)^{-1.5}\right]$

where $r_{\odot}$ is the solar radius. This expression should be a good approximation in the quiet region. For active regions, the background should be ten times larger to compensate for any negative perturbations (Aschwanden 2005). We make some modifications to this formula for our background plasma. Our modified background plasma is given by the expression

$$
\begin{aligned}
n_{\mathrm{bg}}(r)= & 10^{8}\left[10^{5}\left(\frac{r}{r_{\odot}}\right)^{-50}+97.0\left(\frac{r}{r_{\odot}}\right)^{-16}\right. \\
& \left.+28.0\left(\frac{r}{r_{\odot}}\right)^{-6}+0.2\left(\frac{r}{r_{\odot}}\right)^{-1.5}\right] .
\end{aligned}
$$



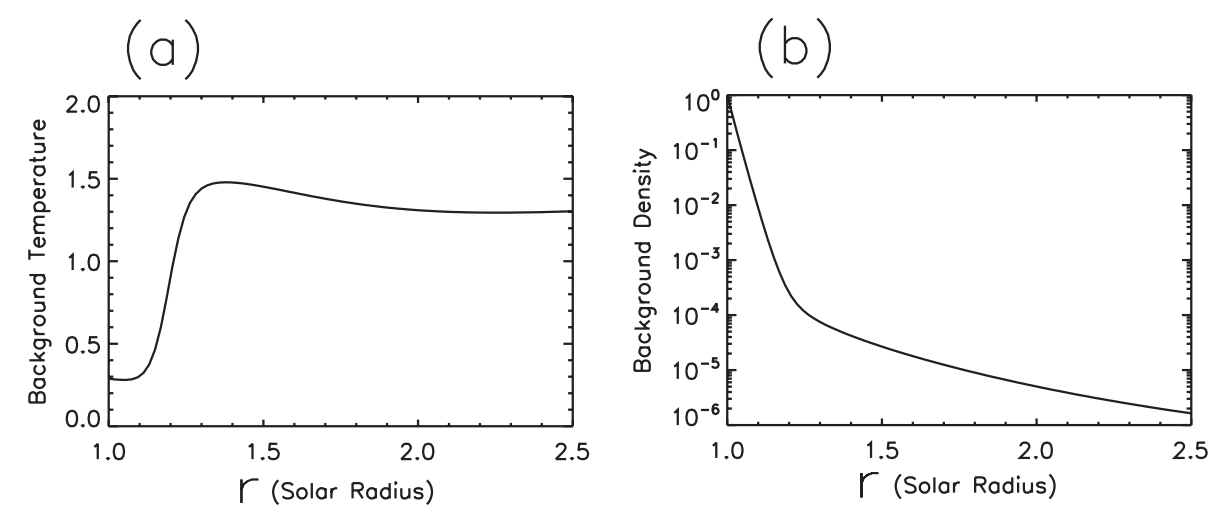

Fig. 3. Background temperature (a)) and density (b)) as a function of radial coordinate in units of the solar radius.
As mentioned above, the strongest perturbation of the plasma density appears in the low corona, so we have to increase our background in the low corona. To take this into account, we add a term to the right hand side, which decreases very rapidly with $r$, so that it does not affect the outer corona. We also change the coefficients for the three other terms to keep the plasma density positive in the outer corona. Through Eqs. $(11,18,20)$, we calculate the background plasma temperature. The stratification of the background temperature and density is given in Fig. 3. From the right panel of the figure, we find the background temperature is in a reasonable range when $r \geq 1.4$. But in the low corona $(r<1.4)$, the background temperature is unreasonably low due to the first term in Eq. (20).

Finally, through Eqs. $(12,13,14)$, we obtain the total plasma pressure, density, and temperature, respectively.

\section{Results}

\subsection{Field lines}

Figure 4 shows the magnetic field lines in the whole corona. Panel a displays the potential field extrapolation, which has no free parameters. Panel $b$ exhibits the result of the LFFF model extrapolation. This model contains the force-free parameter $\alpha$ and we use $\alpha=0.4$. In panel c we show the MHS model field lines. Here we use $\alpha=0.4$ and $a=0.2$. From this figure it is clear that - though the measured photospheric boundary conditions are the same - the different models generated different distributions of the magnetic field in the corona.

\subsection{Current sheet and plasma density in the source surface}

Figure 5 shows the radial component of the magnetic field and the current sheet on the source surface, as obtained from the potential field model (panel a with $\alpha=0$ and $a=0$ ), LFFF model (panel b with $\alpha=0.4$ and $a=0$ ), and MHS model (panel c with $\alpha=0.4$ and $a=0.2$ and panel d with $\alpha=0.6$ and $a=0.2$ ), respectively. The grey shading gives the reversal of the radial component of the magnetic field and the current sheet in the source surface. It is reasonable that the shape of the current sheet is smooth and located near the equatorial plane because it is expected in the solar minimum. But the thickness of the sheet is different between the models. Figure 6 shows the plasma density distribution (normalized by the density in the polar region) at the source surface. It is clear that the density is higher in the current sheet than in the two polar regions. From panel a of Fig. 6, we can see the plasma density in the equatorial plane is twice as high as in the polar region. But in panel b, the density in the equatorial plane is only 1.12 times as high as in the polar region. This difference is due to the different free parameter $a$ in the model.

Note that Figs. 5 and 6 show the radial magnetic field and plasma density on the outer boundary, therefore, the deviation from potential field models is most pronounced. In Fig. 5, the total current density increases with increasing $\alpha$ and $a$. This causes the pinching of the structure. It is important to remember that increasing $a$ also enhances the field-aligned current density and therefore contributes to the narrowing of the structure. Because the plasma magnetic field structure is calculated selfconsistently, stronger Lorentz force has to be balanced by a larger pressure gradient and a higher density. These effects can be seen clearly in the figures. We emphasize that the narrowing in our model is of course not due to solar wind expansion, as shown in Riley et al. (2006)

\subsection{Comparison with the Bogdan-Low-model}

Gibson \& Bagenal (1995) applied the Bogdan-Low-model to a study of the plasma distribution in a dipole field case in the corona. They are successful in constructing the plasma distribution in the corona, especially in the streamer belts, when the corona is approximately longitudinally symmetric. The authors also point out some difficulty applying the Bogdan-Low model directly to data, in particular, using measured photospheric magnetic field directly as boundary conditions (by a spherical harmonic decomposition) is not compatible with a realistic density structure. The problem existed already for solar minimum configurations, but became more severe in the solar activity maximum. The authors find a way around this problem by allowing deviations in the measured and modeled photospheric magnetic field. The resulting configuration describes the large-scale structure reasonable well, but was not able represent structures below a resolution of one tenth of a solar radius.

The Bogdan-Low-model has been extended by Zhao \& Hoeksema (1994) towards the inclusion of horizontal volume and helmet streamer currents. It was demonstrated that this extension allows better modeling of polar plumes and the axes of coronal streamers. Gibson et al. (1996) then applied this extended model to data. Compared to their earlier work, the extended model contains an equatorial current sheet, as well as current sheets between open and closed field regions, as boundary conditions. This allows modeling of the observed sharp gradients across the boundaries of helmet streamers.

The N95 model is the generalization of the Bogdan-Low model to the regime $\alpha \neq 0$. This model has been applied by Zhao et al. (2000) to model the magnetic field structure for polar crown SXR arcades by fitting the free parameters in the N95 model. The authors concentrated mainly on the local 

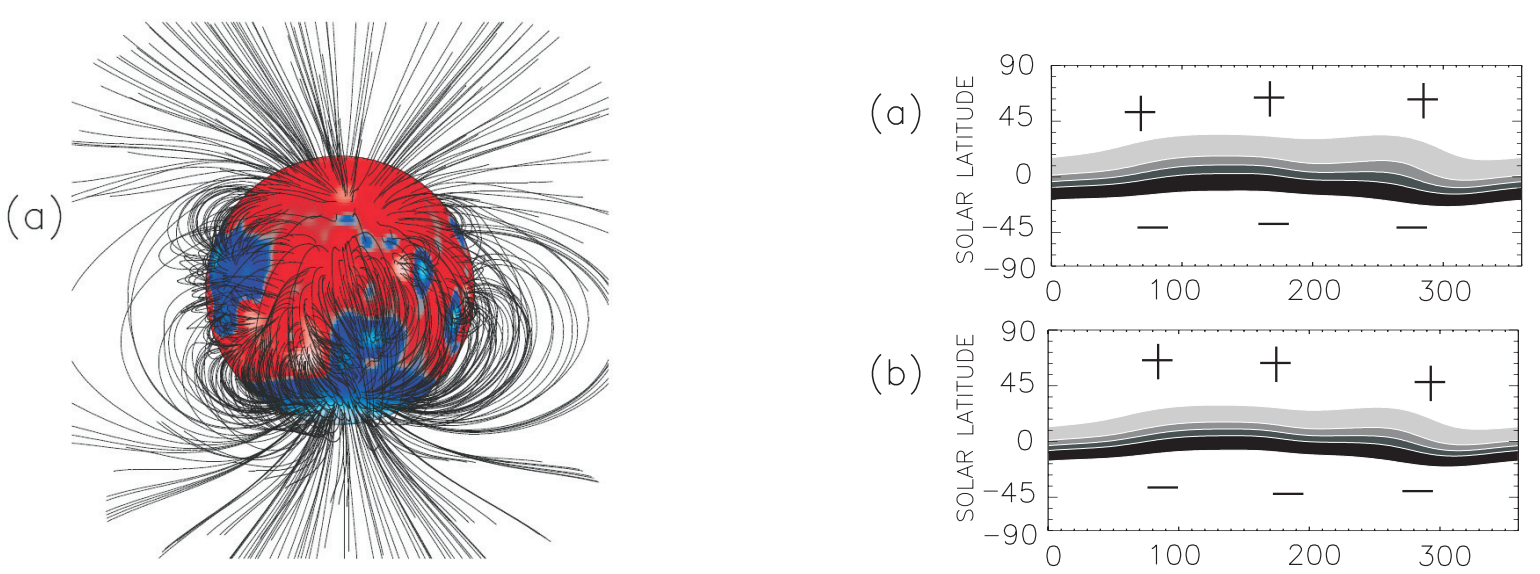

(b)

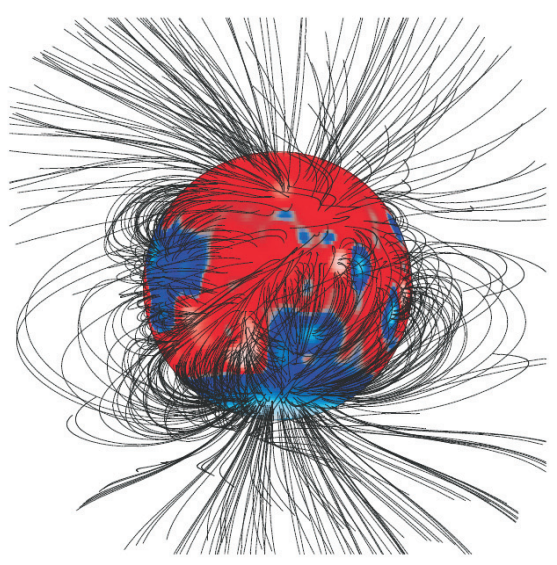

(c)

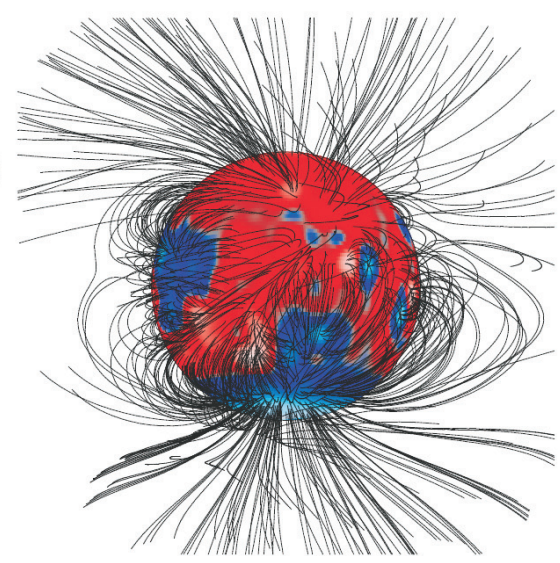

Fig. 4. Magnetic field lines in the corona for Carrington rotation 1919 from different models: a) potential field model, b) LFFF model with $\alpha=0.4$, c) MHS model with $\alpha=0.4$ and $a=0.2$.

configuration over the southern polarity reversal line. Within this paper we applied this more advanced model to constructing the global magnetic field and the plasma in the corona. Our method is also different from that of Gibson \& Bagenal (1995). We start from the magnetogram in the photosphere, whereas Gibson \& Bagenal (1995) use white-light data as primary input for their model.

In the linear force free case, the current density has the form $\mu_{0} \boldsymbol{j}=\alpha \boldsymbol{B}$. This is only applicable in the low $\beta$ case, such as in the low solar corona where the plasma pressure is much lower than the magnetic pressure. In that case we cannot derive any information of the plasma from the model. In this MHS model, (c)

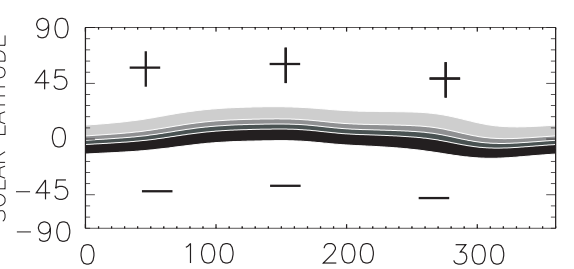

(d)

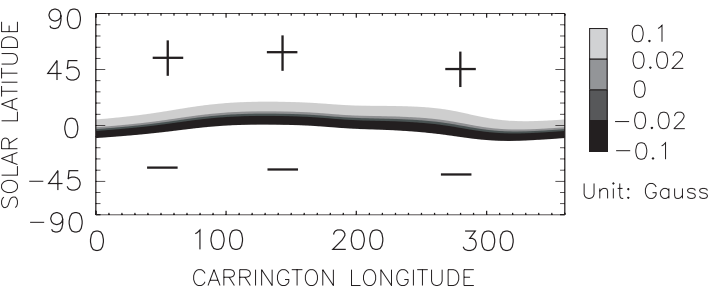

Fig. 5. Current sheet in the source surface from different models: a) the potential field model with $\alpha=0$ and $a=0, \mathbf{b})$ the LFFF model with $\alpha=0.4$ and $a=0, \mathbf{c})$ the MHS model with $\alpha=0.4$ and $a=0.2$, d) the MHS model with $\alpha=0.6$ and $a=0.2$.

(a)

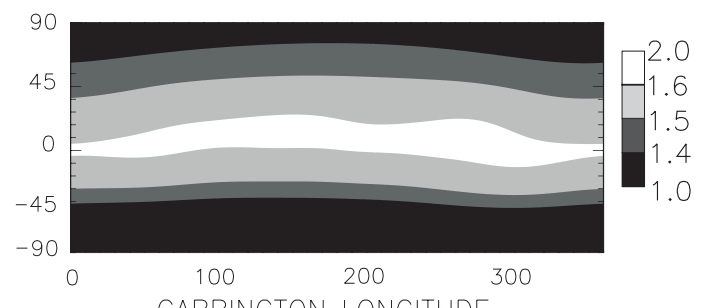

(b)

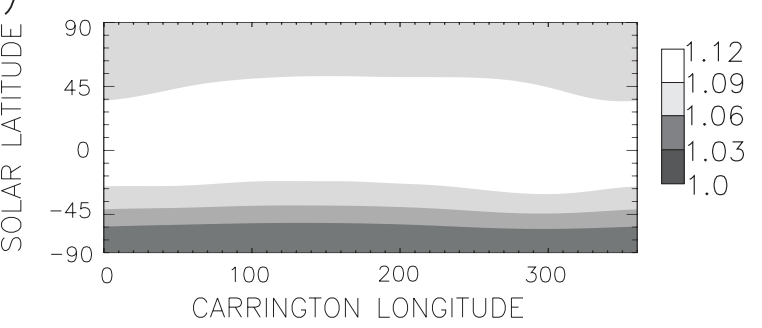

Fig. 6. The density distribution at the source surface (the panel a) with $\alpha=0.4$ and $a=0.2$ and the panel b) with $\alpha=0.4$ and $a=0.05$ ).

there are two components of the current: the pure field-aligned part and the spherical shell part (Fig. 1). They are represented by the first and the second terms in the right side of Eq. (4), 
respectively ${ }^{1}$. The main difference between the linear force-free model and the MHS model is the term $\varepsilon(r) \nabla(\boldsymbol{r} \cdot \boldsymbol{B}) \times \boldsymbol{r}$. This component of the current will produce a Lorentz force that will balance the pressure gradient and the gravity force. Consequently, not only the magnetic field but also the plasma will be constructed from this MHS model self-consistently. These $\alpha$ and $a$ are two free parameters in the model (Eq. (4)). Different free parameters will generate different distributions of the magnetic field and the plasma in the corona. The parameter $\alpha$ is the ratio of the parallel current component to the magnetic field. If $\alpha$ is too large, the magnetic field lines in the corona will become very twisted, which is unreasonable. We vary $\alpha$ from 0 to 0.6. The parameter $a$ represents the perturbation. If $a$ is zero, it is the force-free case and there is no perturbation. When $a$ increases, the perturbation increases. In Figure 6, the difference in the density between the polar region and the equatorial plane is greater with a higher value of $a$. It shows that a larger $a$ generates a stronger perturbation in the model. But we cannot specify $a$ a too large value, because in that case the perturbation is too large and it is impossible to put a reasonable background density to compensate for the perturbation.

\subsection{Influence of free model parameters}

The comparison between the different models (Fig. 4) reveals that the field lines in the polar cap are similar. This is reasonable

because the magnetic field in the polar cap at solar activity minimum should be close to potential. In the low-latitude part in the corona, different models give different results. From Fig. 5, we find that the current sheet in the source surface from different models is very smooth and located close to the equatorial plane. But the thickness of the sheet is different between different models. The current sheet from the MHS model is thinner than the other two models. This should be due to the second component of the current: $\varepsilon(r) \nabla(\boldsymbol{r} \cdot \boldsymbol{B}) \times \boldsymbol{r}$. This term will change the magnetic field distribution in the source surface from the LFFF model, and it reduces the scale of the current sheet. If we keep $a$ constant and increase $\alpha$ from 0.4 to 0.6 , it is clear that the thickness of the current sheet also decreases (panels c and d in Fig. 5). It means that field-aligned current can also reduce the scale of the current sheet on the source surface. Since the MHS model can construct not only the magnetic field but also the plasma in the corona self-consistently, we show the density distribution in the source surface in Fig. 6. From the figure, it is obvious that the density is higher in the equatorial plane than in the two pole regions. This is consistent with the field line distribution in panel $\mathrm{c}$ in Fig. 4. In the equatorial plane, there are many closed field lines that confine the plasma. But in the polar regions, the plasma can escape into the solar wind along the open field lines, so it is reasonable that our MHS model with a current sheet has a higher density in the equatorial plane. It is not obvious from the N95 model equations that the density is reduced in the polar regions and enhanced at the equator.

We also applied our model to the solar activity maximum. The result is not as successful as in the solar minimum. There may be several reasons for this:

- the boundary condition is the photospheric magnetogram synoptic chart. This means we assume that there are no intense changes in the photospheric field during the rotation of

\footnotetext{
${ }_{1}$ Please note that the spherical shell part of the current density also contains a varying component parallel to the field lines, dependent on the local direction of the magnetic field.
}

the sun, but active regions may evolve significantly over one solar rotation;

- another even more important reason may be that, during solar maximum, the solar corona shows a lot more small-scale structures, which are difficult to model with a linear solution such as ours.

Another issue is the first term in Eq. (20). Because the strongest density perturbation always appears in the active regions in the low corona, we have to apply a very high background density to compensate for the perturbation in the low corona. This will bring us an unreasonable density distribution in the low corona. But this does not mean the magnetic field distribution in the low corona is not correct. In our MHS model, we cannot totally avoid the noise. Because the plasma $\beta$ in the low corona is very low, a small angle between the magnetic field line and the current line will produce a very large Lorentz force, which is very hard to be balanced by the plasma.

\section{Conclusions and outlook}

We applied the Neukirch 95 magnetohydrostatic model to reconstructing the magnetic field and the plasma in the solar corona self-consistently with the synoptic photospheric magnetic field observation as the boundary condition. While the overall largescale magnetic field structure is described reasonably well, we encountered some problems regarding the fine structure of the observed photospheric magnetic field being inconsistent with the density distribution under the limitations of the Neukirch 95 model. This problem has already been encountered by Gibson \& Bagenal (1995) who used the simpler Bogdan-Low-model. The additional free parameter $\alpha$ in the Neukirch-model allows a greater variability of possible configurations, but varying $\alpha$ does not resolve this problem.

In general this is to be expected since the models are linear and derive their structure from line-of-sight photospheric magnetic field measurements alone. Since the small-scale coronal structures are likely to be caused by nonlinear effects, further improvements, such as the use of vector magnetograph data, may be necessary to model these structures appropriately.

In the next step, we will develop a more general MHS model to apply to the solar corona. Some basic steps have been taken already with a tomography program developed by Wiegelmann \& Inhester (2003), which provides the 3D plasma density structure more directly, e.g. by using coronagraph images from different viewpoints, as provided by the STEREOmission, for a tomographic inversion. Wiegelmann \& Neukirch (2006) and Wiegelmann et al. (2007) developed codes to solve the MHS-equations (1)-(3) numerically in cartesian and spherical geometry, respectively. The spherical MHS-code generalizes the global nonlinear force-free code developed by Wiegelmann (2007). These numerical programs based on an optimization principle are not limited by the special assumptions (separable solutions) required for the analytic model by Neukirch (1995) used here. The methods are numerically expensive, require the use of parallel computers for reasonable grid resolution, and are also observationally more restrictive because photospheric vector magnetograms are required as input. The necessary full-disc vector magnetograms will become available from the groundbased magnetograph SOLIS and the space-born Solar Dynamics Observatory in the near future.

To summarize, though there are some insufficiencies in this MHS model, the distribution of not only the magnetic field but 
also the plasma in the solar corona can be constructed from this MHS model in a self-consistent way. In the future we will apply a more general MHS model to construct the solar corona.

Acknowledgements. P. Ruan \& L. Feng are supported by the International MaxPlanck Research School on Physical Processes in the Solar System and Beyond at the Universities of Braunschweig and Göttingen. The work of T. Wiegelmann was supported by DLR-grant 50 OC 0501. T. Neukirch acknowledges financial support by the STFC. Financial support by the European Commission through the SOLAIRE Network (MTRN-CT-2006-035484) is also gratefully acknowledged. We acknowledge use of magnetograms from the Wilcox Solar Observatory. We would like to thank the referee, Gordon Petrie, for useful remark that improve this paper.

\section{References}

Aly, J. J. 1989, Sol. Phys., 120, 19

Amari, T., Aly, J. J., Luciani, J. F., Boulmezaoud, T. Z., \& Mikic, Z. 1997, Sol. Phys., 174, 129

Amari, T., Boulmezaoud, T. Z., \& Aly, J. J. 2006, A\&A, 446, 691

Antiochos, S. K., DeVore, C. R., \& Klimchuk, J. A. 1999, ApJ, 510, 485

Aschwanden, M. J. 2005, Sol. Phys., 228, 339

Aschwanden, M. J., Nightingale, R. W., \& Alexander, D. 2000, ApJ, 541, 1059

Aulanier, G., \& Demoulin, P. 1998, A\&A, 329, 1125

Aulanier, G., Schmieder, B., van Driel-Gesztelyi, L., et al. 2000, Adv. Space Res., 26, 485

Bogdan, T. J., \& Low, B. C. 1986, ApJ, 306, 271

Carcedo, L., Brown, D. S., Hood, A. W., Neukirch, T., \& Wiegelmann, T. 2003, Sol. Phys., 218, 29

Chiu, Y. T., \& Hilton, H. H. 1977, ApJ, 212, 873

Cuperman, S., Detman, T. R., Bruma, C., \& Dryer, M. 1992, A\&A, 265, 785

Feng, L., Wiegelmann, T., Inhester, B., et al. 2007, Sol. Phys., 241, 235

Gibson, S. E., \& Bagenal, F. 1995, J. Geophys. Res., 100, 19865

Gibson, S. E., Bagenal, F., \& Low, B. C. 1996, J. Geophys. Res., 101, 4813

Hoeksema, J. T. 1991, Adv. Space Res., 11, 15

Inhester, B., \& Wiegelmann, T. 2006, Sol. Phys., 235, 201

Kano, R., \& Tsuneta, S. 1995, ApJ, 454, 934

Krivova, N. A., \& Solanki, S. K. 2004, A\&A, 417, 1125

Lagg, A. 2005, in Chromospheric and Coronal Magnetic Fields, ed. D. E. Innes, A. Lagg, \& S. A. Solanki, ESA SP-596

Low, B. C. 1985, ApJ, 293, 31

Low, B. C. 1991, ApJ, 370, 427

Low, B. C. 1992, ApJ, 399, 300

Low, B. C. 1993a, ApJ, 408, 689

Low, B. C. 1993b, ApJ, 408, 693
Marsch, E., Wiegelmann, T., \& Xia, L. D. 2004, A\&A, 428, 629

Metcalf, T. R., DeRosa, M. L., Schrijver, C. J., et al. 2008, solphys, 247, 269

Mikić, Z., Linker, J. A., Schnack, D. D., Lionello, R., \& Tarditi, A. 1999, Phys. Plasmas, 6, 2217

Neukirch, T. 1995, A\&A, 301, 628

Neukirch, T. 1997, A\&A, 325, 847

Neukirch, T., \& Rastätter, L. 1999, A\&A, 348, 1000

Petrie, G. J. D., \& Neukirch, T. 2000, A\&A, 356, 735

Pneuman, G. W., \& Kopp, R. A. 1971, Sol. Phys., 18, 258

Riley, P., Linker, J. A., Mikić, Z., et al. 2006, ApJ, 653, 1510

Rosner, R., Tucker, W. H., \& Vaiana, G. S. 1978, ApJ, 220, 643

Rudenko, G. V. 2001, Sol. Phys., 198, 279

Sakurai, T. 1989, Space Sci. Rev., 51, 11

Schmidt, H. U. 1964, in the Physics of Solar Flares, 107

Schrijver, C. J., Sandman, A. W., Aschwanden, M. J., \& DeRosa, M. L. 2004, ApJ, 615, 512

Schrijver, C. J., DeRosa, M. L., Title, A. M., \& Metcalf, T. R. 2005, ApJ, 628, 501

Schrijver, C. J., Derosa, M. L., Metcalf, T. R., et al. 2006, Sol. Phys., 235, 161

Schwenn, R., Inhester, B., Plunkett, S. P., et al. 1997, Sol. Phys., 175, 667

Seehafer, N. 1978, Sol. Phys., 58, 215

Semel, M. 1967, Annales d'Astrophysique, 30, 513

Serio, S., Peres, G., Vaiana, G. S., Golub, L., \& Rosner, R. 1981, ApJ, 243, 288

Solanki, S. K., Lagg, A., Woch, J., Krupp, N., \& Collados, M. 2003, Nature, 425, 692

Wheatland, M. S., Sturrock, P. A., \& Roumeliotis, G. 2000, ApJ, 540, 1150

White, S. M. 2002, Astron. Nachr., 323, 265

Wiegelmann, T. 2004, Sol. Phys., 219, 87

Wiegelmann, T. 2007, Sol. Phys., 240, 227

Wiegelmann, T. 2008, JGR, 113, 3

Wiegelmann, T., \& Inhester, B. 2003, Sol. Phys., 214, 287

Wiegelmann, T., \& Inhester, B. 2006, Sol. Phys., 236, 25

Wiegelmann, T., \& Neukirch, T. 2002, Sol. Phys., 208, 233

Wiegelmann, T., \& Neukirch, T. 2006, A\&A, 457, 1053

Wiegelmann, T., Schindler, K., \& Neukirch, T. 1998, Sol. Phys., 180, 439

Wiegelmann, T., Schindler, K., \& Neukirch, T. 2000, Sol. Phys., 191, 391

Wiegelmann, T., Inhester, B., Lagg, A., \& Solanki, S. K. 2005a, Sol. Phys., 228, 67

Wiegelmann, T., Lagg, A., Solanki, S. K., Inhester, B., \& Woch, J. 2005b, A\&A, 433, 701

Wiegelmann, T., Neukirch, T., Ruan, B., \& Inhester, B. 2007, A\&A, 475, 701

Wu, S. T., Guo, W. P., Andrews, M. D., et al. 1997, Sol. Phys., 175, 719

Wu, S. T., Guo, W. P., \& Wang, J. F. 1995, Sol. Phys., 157, 325

Zhao, X., \& Hoeksema, J. T. 1993, Sol. Phys., 143, 41

Zhao, X., \& Hoeksema, J. T. 1994, Sol. Phys., 151, 91

Zhao, X. P., Hoeksema, J. T., \& Scherrer, P. H. 2000, ApJ, 538, 932 